\title{
Treatment of Invasive Mucormycosis with Intravenous Isavuconazonium and Topical Amphotericin B in a Renal-Impaired Patient: Case Report and Review of the Literature
}

\author{
Alvaro F. Galvez, ' Ozana L. Lipka, Linwood R. Haith,' Dane Scantling, \\ Mark A. Kaplan, Mary Lou Patton, and Robert E. Guilday²
}

\begin{abstract}
Background: Burn patients are a high-risk population for development of opportunistic infections. Patients suffering extensive burns require prolonged hospital stays and often endure multiple infections from multiple drug-resistant organisms (MDRO). In view of the emergence of MDRO, newer less toxic antimicrobial alternatives are sought for better care of these patients. Treatment of severe opportunistic mycoses in burn patients have proved challenging because of the commonly recommended treatment with amphotericin $\mathrm{B}$, a toxic polyene anti-fungal agent.

Methods: A novel treatment modality was utilized on one patient with acute renal failure in our regional burn center. Systemic isavuconazonium was used with topical amphotericin B in lieu of the standard therapy of systemic amphotericin B.

Results: The patient remained hemodynamically stable and underwent several successful skin grafts to residual open wounds of her head, chest, back, abdomen, and upper extremities. Tissue biopsies during subsequent grafting did not show Mucor growth since therapy initiation. Her renal function recovered completely and renal replacement therapy was discontinued.

Conclusions: Dual therapy with systemic isavuconazonium and topical amphotericin B is a viable alternative to treat invasive mucormycosis in burn patients with comorbidities, including acute renal injury (AKI), that prohibit use of amphotericin B systemically.
\end{abstract}

Keywords: burns; critical care; surgical infection; trauma

B URN PATIENTS lose the epithelial barrier that provides protection from infection from otherwise saprophytic micro-organisms inhabiting human integumentary and visceral surfaces as well as the environment. This places them at higher risk than any other hospitalized patient for opportunistic infections [1]. These iatrogenic infections are an important cause of later-stage morbidity and mortality in patients with extensive burns, who invariably are immunosuppressed. These patients typically have been exposed to polymicrobial antibiotics, which eliminate commensal flora. This promotes overgrowth of opportunistic species, including fungi, that may evolve from simple colonization to invasive infection through the burn wound. Systemic antifungal treatment may be required and often the only alternative is the potentially nephrotoxic amphotericin B. We present the case of a renally impaired, extensively burned patient who developed invasive mucormycosis treated successfully with intravenous isavuconazonium sulfate $\left(\mathrm{Cresemba}^{\circledR}\right.$, Astellas

\footnotetext{
${ }^{1}$ Department of General Surgery, Drexel University College of Medicine/Hahnemann University Hospital, Philadelphia, Pennsylvania.

${ }^{2}$ Nathan Speare Regional Burn Treatment Center, Crozer Chester Medical Center, Upland, Pennsylvania.

(C) Alvaro F. Galvez et al. 2017; Published by Mary Ann Liebert, Inc. This Open Access article is distributed under the terms of the Creative Commons License (http://creativecommons.org/licenses/by/4.0), which permits unrestricted use, distribution, and reproduction in any medium, provided the original work is properly credited.
} 
Pharma Inc., Tokyo, Japan) and topical amphotericin B soaks. We present this alternative as a novel anti-fungal treatment for invasive mycosis in a patient precluded from receiving amphotericin $B$ because of evolving acute renal injury (AKI).

\section{Case Presentation}

We present the case of a 58-year-old female involved in a house fire who was transferred to our institution after being intubated for inhalation injury and stabilized at an outside hospital. Initial assessment revealed $47 \%$ total body surface area (TBSA) burns with multiple deep partial thickness and full thickness burns involving most of her face, neck, upper extremities, and upper anterior and posterior torso.

Her past medical history was significant for obesity with an admission body mass index (BMI) of 35, active tobacco use, chronic obstructive pulmonary disease (COPD), and three prior cerebrovascular accidents with residual left-sided weakness. Bilateral upper extremity escharotomies were performed on admission for full thickness circumferential burns.

Initial laboratory work was significant for a creatinine of 1.6 and a blood urea nitrogen (BUN) of 15 , with a creatinine kinase (CK) of $4703 \mathrm{U} / \mathrm{L}$. During the first hours of admission the urine output decreased consistently and potassium concentrations reached $>6 \mathrm{mEq} / \mathrm{L}$ with a $\mathrm{pH}$ decrease to $<7.20$. The patient was diagnosed with AKI and continuous renal replacement therapy (CRRT) was initiated.

Bronchoscopy confirmed inhalation injury. Empiric broadspectrum antibiotic coverage with vancomycin and piperacillin/tazobactam (Zosyn ${ }^{\circledR}$, Wyeth Pharmaceuticals Inc., a subsidiary of Pfizer Inc., Philadelphia, PA) was started on the first post-burn day given her extensive TBSA burns, fevers, leukocytosis, and developing multi-organ failure.

On the eighth post-burn day the patient was stabilized enough to be taken to the operating room for excision of torso and bilateral upper extremities burn wounds. Thereafter, the patient remained stable and underwent several procedures for excision and split-thickness skin grafting of open wounds.
Additionally, several digital amputations, tracheostomy, and percutaneous endoscopic gastrostomy (PEG) tube placement were performed.

Several superficial wound culture swabs were obtained, which speciated bacteria that included methicillin-sensitive Staphylococcus aureus (MSSA), Escherichia coli, several strains of increasingly resistant Pseudomonas aeruginosa, vancomycin-resistant Enterococcus casseliflavus (VRE), Chryseomonas luteola, Stenotrophomonas maltophilia, Achromobacter xyloxidans, Serratia plymuthica, Enterobacter cloacae, and unspecified Mucor species. The patient required several antibiotics during her hospital stay including cefazolin (Ancef ${ }^{\circledR}$, GlaxoSmithKline, Research Triangle Park, NC), vancomycin $\left(\right.$ Vancocin $\left.{ }^{\circledR}\right)$, metronidazole (Flagyl ${ }^{\circledR}$, Pfizer Inc., New York, NY), piperacillintazobactam (Zosyn), levofloxacin (Levaquin ${ }^{(}$, Janssen Pharmaceutica, Beerse, Belgium), and imipenem-cilastatin (Primaxin ${ }^{\circledR}$, Merck, Kenilworth, NJ). The patient was also treated with anti-fungal medications, which included fluconazole and micafungin for Candida spp.

On hospital day 19, the patient developed crusty plaquelike growths of a foul-smelling yellowish eschar on her upper torso (Fig. 1 and 2). This was accompanied by development of hypotension, tachycardia, intermittent fever episodes, and a leukocytosis exceeding 20,000 cells per microliter. Specimens of these exophytic lesions were sent to pathology as well as microbiology. Results yielded growth of Mucor spp. and histologic examination revealed pathognomonic hyphal structures invading subcutaneous adipose tissue (Figs. 3-5). Based on recent literature that corroborates achievement of therapeutic dermal concentrations of topically applied amphotericin B, dual therapy was initiated with amphotericin B deoxycholate soaks on the burn wound dressings as well as intravenous liposomal amphotericin B (AmBisome ${ }^{\circledR}$, Gilead, Foster City, CA) [2].

Approximately $72 \mathrm{~h}$ after initiation of anti-fungal treatment the patient's vasopressor requirements decreased and the white blood cell count and bandemia initiated a

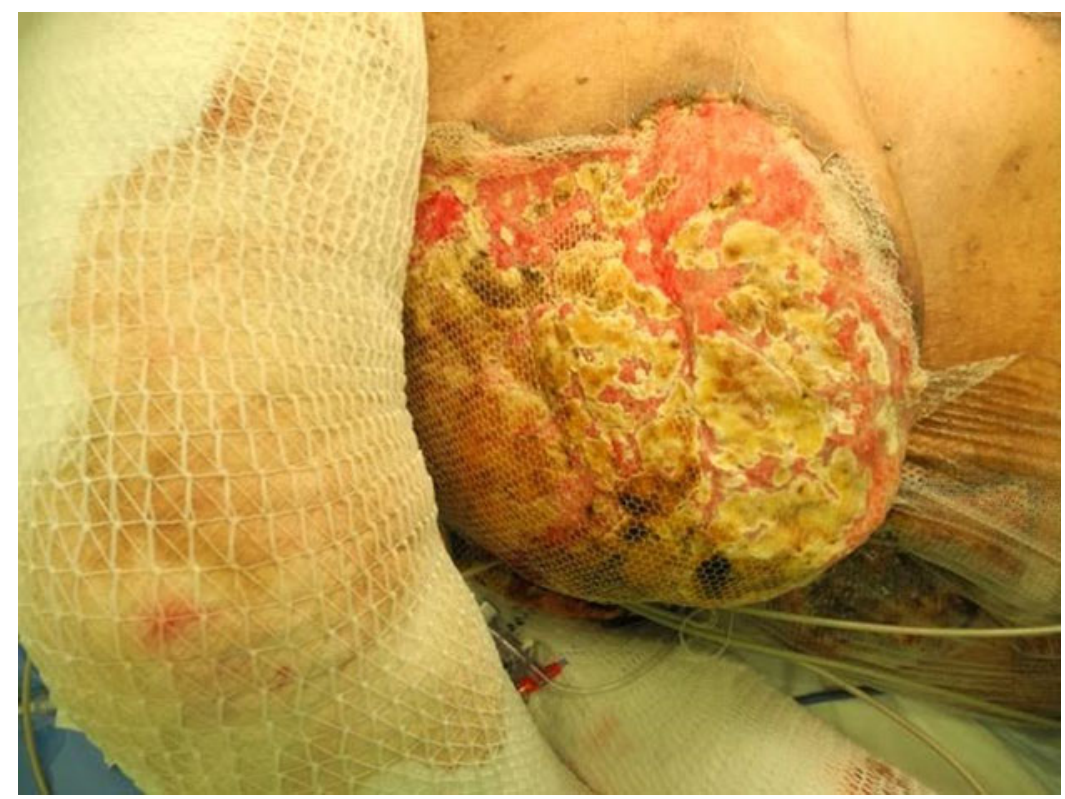

FIG. 1. Plaque-like growth of Mucor on the patient's right breast. 


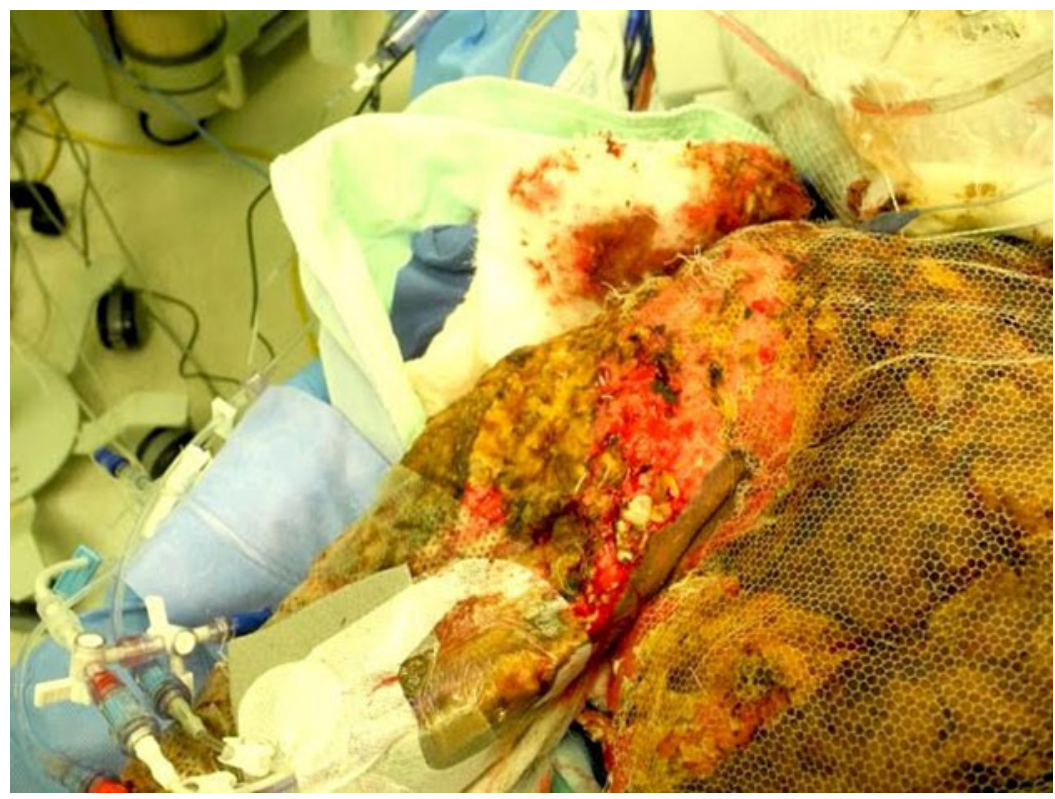

FIG. 2. Plaque-like growth of Mucor on patient right chest wall and deltoid area

downward trend. At this point the patient's evidence of improvement in renal function had been demonstrated by the spontaneous production of urine, although she remained dependent on renal replacement therapy (RRT). Given this improving renal function an alternative to AmBisome was considered in an effort to support renal recovery. Isavuconazonium was chosen, based on recent literature supporting its successful use in other invasive mucormycoses [3-5].

The dosing currently utilized for isavuconazonium sulfate was $372 \mathrm{mg}$ intravenous (IV) daily, after a loading dose of $372 \mathrm{mg}$ IV and for the first $48 \mathrm{~h} 372 \mathrm{mg}$ IV every eight hours (see below), along with amphotericin B soaks applied every eight hours to the remaining open wounds at a dilution of $24 \mathrm{mcg} / \mathrm{mL}$ (24 mg in $1000 \mathrm{~mL}$ of sterile water) [2,6].
The patient remained hemodynamically stable and underwent several successful skin grafts to residual open wounds of her head, chest, back, abdomen and upper extremities. The fungal lesions observed on her chest wall. Several tissue biopsies during subsequent grafting have failed to show Mucor growth since initiating therapy. Her renal function recovered completely and renal replacement therapy was discontinued.

A percutaneous gastrostomy (PEG) tube had been performed for nutritional access early in the patient's hospital course, and although Cresemba was available in enteral formulation, the capsule proved too large to be administered through the PEG tube, therefore, the patient was changed to posaconazole (see below), available in suspension from.

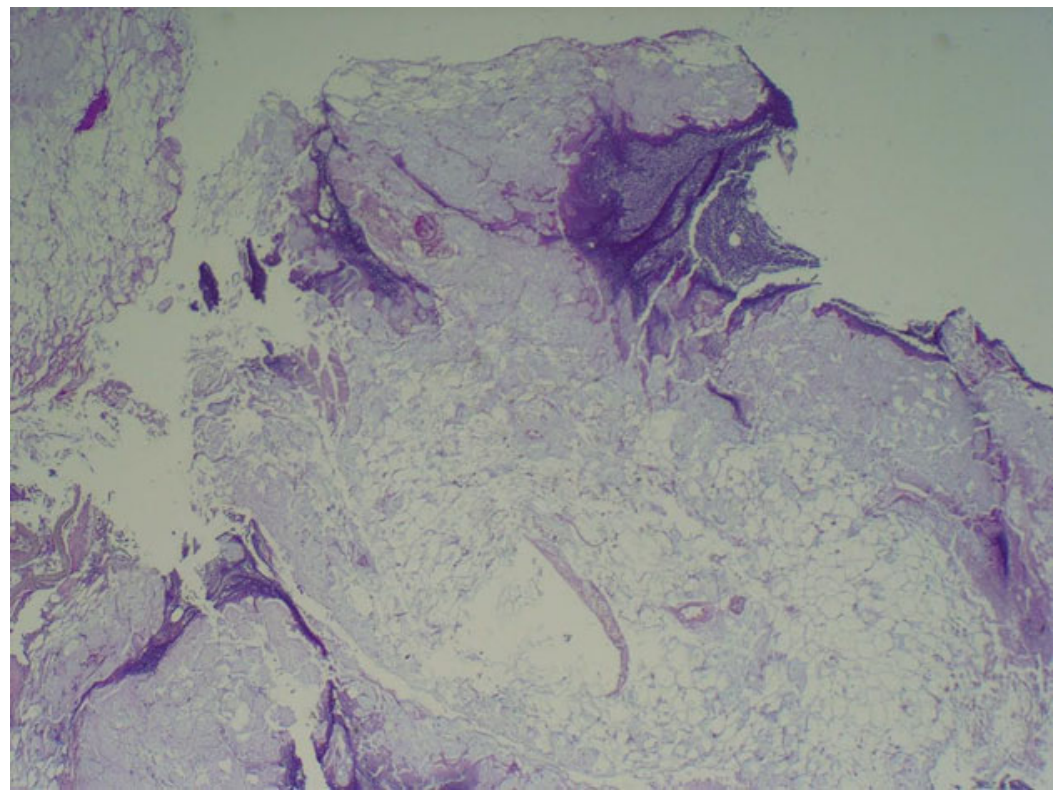

FIG. 3. Tissue section showing partial necrosis and inflammatory reaction involving subcutaneous adipose tissue. 


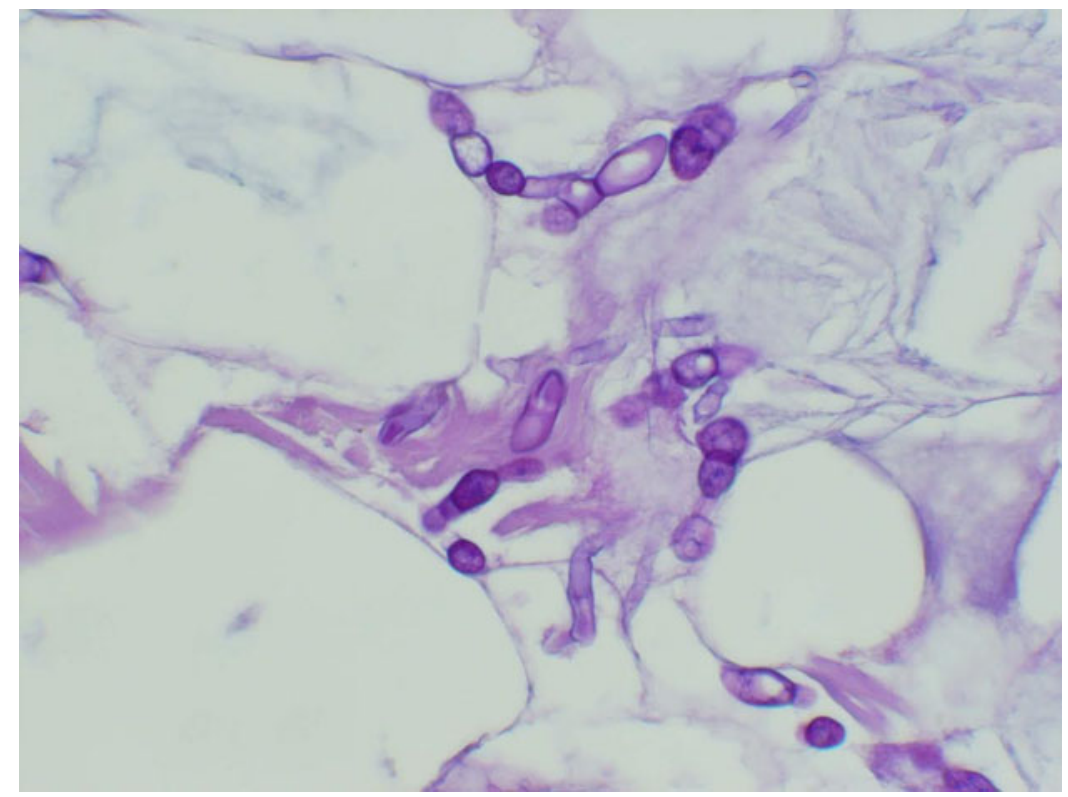

FIG. 4. Periodic acid-Schiff (PAS) stain highlighting the presence of broad-based, irregularly branching hyphae characteristic of Mucor within the subcutaneous adipose tissue at high power.

\section{Discussion}

Burn wound infections are caused by bacteria $(70 \%)$, followed by fungi $(20 \%-25 \%)$, and rarely viruses $(5 \%-10 \%)$ [7]. Fungemia was initially reported in the 1960s, when both

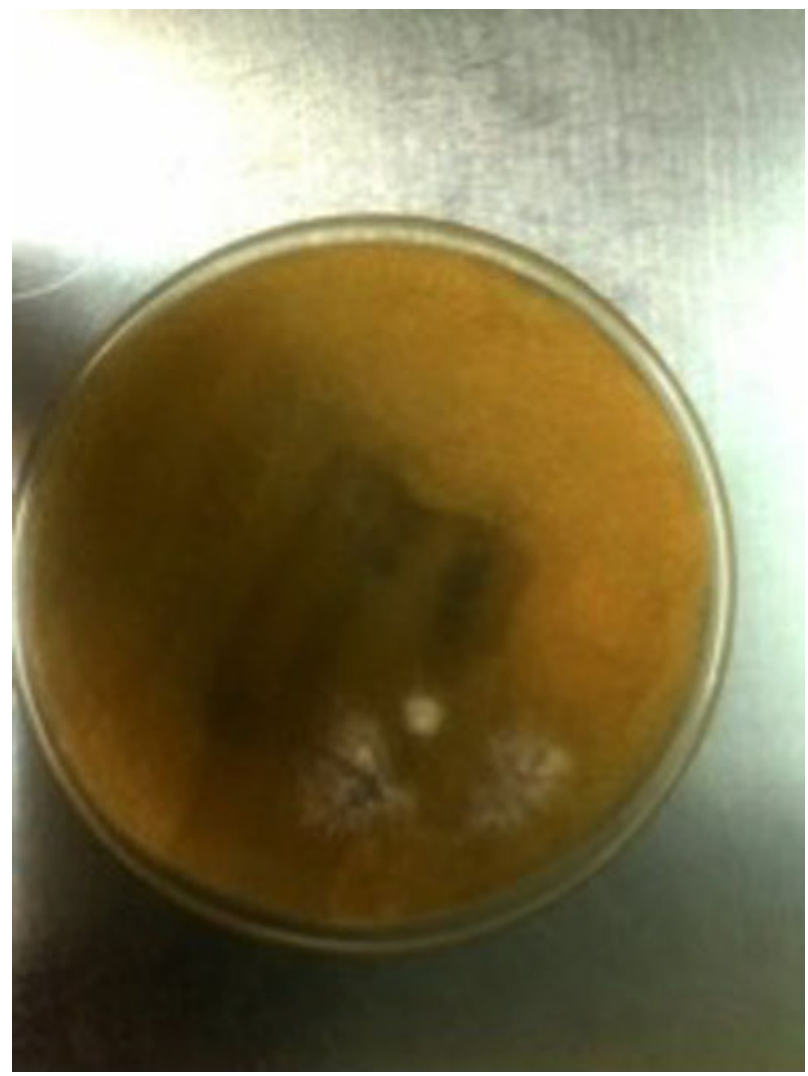

FIG. 5. Mucor species growth on Sabouraud agar. topical and systemic antibiotic treatments were used on burns. Currently, fungal infections occur typically after systemic treatment with polymicrobial antibiotics, which eradicate commensal flora that in normal circumstances might check fungal superinfection [8]. It is critical to diagnose and initiate prompt treatment of invasive fungal disease as mortality can exceed $30 \%$ with delayed treatment [9]. Even with prompt anti-fungal initiation, development of resistant nonalbicans Candida spp., Aspergillus, and Zygomycetes has been well reported and characterized $[10,11]$.

The Mucor species comprises six molds that are ubiquitously found in soil. Human zygomycosis caused by mucorales occur exclusively as opportunistic infection [7]. Risk factors that predispose to this infection include type 1 diabetes mellitus, iron chelation therapy, neutropenia, sustained immunosuppressive therapy, broad spectrum antibiotic use, and open burn wounds [20]. Burn stress may promote pseudo-diabetes with persistent hyperglycemia and glucosuria producing ketotic acidosis. Rhizopus species have an active ketone reductase system enabling proliferation in an acidic $\mathrm{pH}$. They also show increased growth in iron-rich environments, which are promoted in acidosis where serum iron binding capacity is decreased and iron ions are more available for the fungal cells. Hyperglycemia also enhances fungal growth and acidosis impairs neutrophil chemotaxis. Consequently, invasive mucormycosis is a condition frequently described in patients with type 1 diabetes mellitus with diabetic ketoacidosis [12-14].

As a result of the well-known difficulties of fungal culture latency and differentiation between colonization and infection compared with bacteria, there should be a low threshold for obtaining tissue biopsies. Appearance of burn wounds should prompt the clinician to send at least three different biopsies to observe hyphae invading into tissue as this is the most certain method to diagnose invasive fungal infection $[13,15]$. 
Treatment involves aggressive surgical debridement and prompt intravenous anti-fungal therapy; amphotericin B for zygomycetes in particular given the high rate of resistance to commonly used anti-fungal agents [3,8]. Amphotericin B is bactericidal to most pathogenic fungi in humans but approximately $30 \%-40 \%$ of patients suffer AKI that requires hemodialysis [16]. Specialized lipid preparations have been developed to enhance binding to fungal cell membranes and reduce binding to mammalian cells, reducing the risk of renal injury. The most commonly used is the liposomal preparation of amphotericin B (AmBisome), which reduces the risk of renal damage to $15 \%$ [17]. Less toxic alternatives reported in the literature include posaconazole and isavuconazole.

Recent reports have suggested posaconazole (a secondgeneration azole anti-fungal agent) as a viable alternative to amphotericin B-intolerant non-burn patients with invasive mucormycosis $[18,19]$. Isavuconazonium sulfate (Cresemba) has also been reported to be effective in the treatment of nonburn wound mucormycosis refractory to amphotericin B and posaconazole [3]. In March 2015 the Food and Drug Administration (FDA) approved Isavuconazole for the use of invasive mucormycosis, although an extensive review of the literature prior to this publication has not shown any experience with its use in burn patients [4].

Isavuconazonium sulfate (Cresemba) is the prodrug of isavuconazole that allows rapid oral or intravenous delivery. It is hydrolyzed quickly by serum esterases into isavuconazole, a triazole anti-fungal agent that inhibits lanosterol 14 alpha-demethylase that converts lanosterol to ergosterol. The resulting depletion of ergosterol compromises fungal cell membrane synthesis. A loading dose of $372 \mathrm{mg}$ isavuconazonium sulfate (equivalent to $200 \mathrm{mg}$ of isavuconazole) every eight hours for six doses is required to achieve therapeutic blood concentrations. This dosing is then changed to a maintenance dose of $372 \mathrm{mg}$ isavuconazonium sulfate once daily via oral or IV administration [6].

Coverage of the burn area by grafting or flaps is the only therapy that will prevent recurrence in burn wound infection, and it should be performed only after all clinical evidence of sepsis is resolved and the wound infection is controlled, documented by serial surveillance negative swab cultures and biopsies $[9,13]$.

Fungal burn wound infections continue to increase as treatment for extensive burn wound injuries improves and mortality rates in these patients decrease. Anti-fungal resistance is becoming a more frequent phenomenon and newer and less toxic alternatives to salvage anti-fungal treatment are necessary.

As assessed by the favorable response to dual therapy with systemic isavuconazonium and topical amphotericin B we recommend this combination as an option to treat invasive mucormycosis in burn patients with comorbidities, especially AKI, precluding amphotericin use. As with any case report, additional studies with burn patients may be required to establish this alternative as the standard of care.

\section{Acknowledgments}

Written informed consent was obtained from the patient's power of attorney for the publication of this case report and any accompanying images. The authors have no commercial associations to disclose. All images are original and created by the authors.

\section{Author Disclosure Statement}

No competing financial interests exist.

\section{References}

1. Fridkin SK, Jarvis WR. Epidemiology of nosocomial fungal infections. Clin Microbiol Rev 1996;9:499-511.

2. Perez AP, Altube MJ, Schillreff P, et al. Topical amphotericin $\mathrm{B}$ in ultradeformable liposomes: Formulation, skin penetration study, antifungal and antileishmanial activity in vitro. Colloids Surf Biointerfaces 2016;139:190-198.

3. Ervens J, Gahnnoum M, Graf B, Schwartz S. Successful isavuconazole salvage therapy in a patient with invasive mucormycosis. Infection 2014;42:429-432.

4. National Institutes of Health. Isavuconazole in the treatment of renally impaired aspergillosis and rare fungi. NLM identifier: NCT00634049. ClinicalTrials.gov. www.clinicaltrials .gov/ct2/show/NCT00634049 (Last accessed June 13, 2016).

5. Kovanda LL, Desai AV, Lu Q, et al. Isavuconazole Population pharmacokinetic analysis using non-parametric estimation in patients with invasive fungal disease: Results from the VITAL Study. Antimicrob Agents Chemother 2016;60: 4568-4576.

6. Cresemba (isovuconazole sulfate) [prescribing information]. Astella Pharma US, Inc. www.astellas.us/docs/ cresemba.pdf (Last accessed June 14, 2016).

7. Horvath EE, Murray CK, Vaughan GM, et al. Fungal wound infection (not colonization) is independently associated with mortality in burn patients. Ann Surg 2007;245: 978-985.

8. Church D, Elsayed S, Reid O, et al. Burn wound infections. Clin Microbiol Rev 2006;19:403-434.

9. Murray CK, Loo FL, Hospenthal DR, et al. Incidence of systemic fungal infection and related mortality following severe burns. Burns 2008;34:1108-1112.

10. Chakrabarti A, Chatterjee SS, Rao KL, et al. Recent experience with fungaemia: Change in species distribution and azole resistance. Scand J Infect Dis 2009;41:275-284.

11. Mathew BP, Nath M. Recent approaches to antifungal therapy for invasive mycoses. ChemMedChem 2009;4: 310-323.

12. Spellberg B, Edwards J, Ibrahim A. Novel perspectives on mucormycosis: Pathophysiology, presentation, and management. Clin Microbiol Rev 2005;18:556-569.

13. Capoor MR, Sarabahi S, Tiwari VK, Narayanan RP. Fungal infections in burns: Diagnosis and management. Indian $\mathbf{J}$ Plast Surg 2010;43(suppl):S37-S42.

14. Ribes JA, Vanover-Sams CL, Baker DJ. Zygomycetes in human disease. Clin Microbiol Rev 2000;13:236-301.

15. Struck MF, Gille J. Fungal infections in burns: A comprehensive review. Ann Burns Fire Disasters 2013;26:147-153.

16. Wingard JR, Kubilis P, Lee L, et al. Clinical significance of nephrotoxicity in patients treated with amphotericin B for suspected or proven aspergillosis. Clin Infect Dis 1999;29: 1402-1407.

17. Wingard JR, White MH, Anaissie E, et al. A randomized, double-blind comparative trial evaluating the safety of liposomal amphotericin B versus amphotericin B lipid complex in the empirical treatment of febrile neutropenia. Clin Infect Dis 2000;31:1155-1163.

18. Tarani L, Costantino F, Notheis G, et al. Long term posaconazole treatment and follow-up of rhino-orbital-cerebral mucormycosis in a diabetic girl. Pediatr Diabetes 2009;10: 289-293. 
19. Kulendra K, Habibi M, Butler C, et al. Use of posaconazole in the treatment of infective rhinocerebral mucormycosis. J Laryngol Otol 2010;124:1314-1317.

Address correspondence to: Dr. Alvaro Galvez 231 North Broad Street Philadelphia, PA 19102

E-mail: alvarofxgalvez@gmail.com

$\begin{aligned} & \text { Abbreviations Used } \\ \mathrm{AKI} & =\text { acute renal injury } \\ \mathrm{BMI} & =\text { body mass index } \\ \mathrm{BUN} & =\text { blood urea nitrogen } \\ \mathrm{CK} & =\text { creatinine kinase } \\ \mathrm{COPD} & =\text { chronic obstructive pulmonary disease } \\ \mathrm{CRRT} & =\text { continuous renal replacement therapy } \\ \mathrm{FDA} & =\text { Food and Drug Administration }\end{aligned}$

$\begin{aligned} \mathrm{IV}= & \text { intravenous } \\ \mathrm{MDRO}= & \text { multiple drug-resistant organisms } \\ \mathrm{MSSA}= & \text { methicillin-sensitive Staphylococcus } \\ & \text { aureus } \\ \mathrm{RRT}= & \text { renal replacement therapy } \\ \mathrm{PAS}= & \text { periodic acid-Schiff } \\ \mathrm{PEG}= & \text { percutaneous endoscopic gastrostomy } \\ \mathrm{TBSA}= & \text { total body surface area } \\ \mathrm{VRE}= & \text { vancomycin-resistant Enterococcus } \\ & \text { casseliflavus }\end{aligned}$

Cite this article as: Galvez A, Lipka O, Haith L, Scantling D, Kaplan M, Patton ML, Guilday R (2017) Treatment of invasive mucormycosis with intravenous isavuconazonium and topical amphotericin B in a renal impaired patient: Case report and review of the literature. Surgical Infections Case Reports 2:1, 40-45, DOI: 10.1089/crsi.2017.0007 